\title{
Understanding Post-Spaceflight Orthostatic Intolerance - a Simulation Study
}

\author{
T Heldt, RG Mark
}

\author{
Massachusetts Institute of Technology, Cambridge, MA, USA
}

\begin{abstract}
We have developed a computational model of the cardiovascular system with the aim of analyzing the hemodynamic response to orthostatic stress. Using the model, we investigate the hemodynamic effects of changes in model parameters that are thought to contribute to the syndrome of post-spaceflight orthostatic intolerance. Our simulations indicate that changes in total blood volume have the largest detrimental effect on arterial blood pressure homeostasis. If the baseline volume status is borderline hypovolemic, changes in other parameters can significantly impact the system's ability to maintain blood pressure in the upright posture. In particular, any deleterious change in the venous tone feedback impairs blood pressure homeostasis significantly. This result has important implications for countermeasure development as it suggest that an $\alpha$-adrenergic agonist might alleviate the orthostatic syndrome seen post-flight.
\end{abstract}

\section{Introduction}

Human space exploration has demonstrated that the cardiovascular system adapts quickly to the weightless environment and thus enables astronauts to perform well in space with essentially normal hemodynamic status. While such adaptive changes are entirely appropriate in space, they seriously impair cardiovascular performance upon return to the normal gravitational environment as evidenced by the high incidence of post-spaceflight orthostatic intolerance (OI): after short-duration (Shuttle) missions up to $64 \%$ of astronauts fail to complete a 10 -minute stand test on landing day [1]. The incidence jumps to over $80 \%$ after long-duration (MIR or International Space Station) missions [2]. Although considerable experimental effort has been focused on this problem over the past forty years, there remains a critical lack of understanding as to its physiological causes. Mechanisms that have been proposed to contribute to OI include hypovolemia, cardiac atrophy, autonomic dysfunction, and changes in the venous compliance of the legs (see [3] for a recent review).

While each proposed mechanism offers a plausible explanation for the genesis of post-spaceflight OI, it is im- perative to understand the relative importance of each of these mechanisms in order to aid in the development of a functional countermeasure. Given the small number of astronauts studied on each mission, it is unlikely that experimental methods can be used to establish a hierarchy of importance among the proposed mechanisms.

The goal of this paper is to analyze, in the context of a computational model, the impact each of the proposed mechanisms has on the system-level cardiovascular response to orthostatic stress. In so doing, we generate insight into the relative importance of these mechanisms, which in turn enables us to suggest a possible countermeasure to alleviate the orthostatic symptoms seen post-flight.

\section{Cardiovascular model}

We have developed a computational model consisting of a lumped-parameter representation of the hemodynamic system coupled to set-point models of the reflex pathways responsible for short-term blood pressure control [4].

For the hemodynamic model, we subdivided the human vasculature into 17 compartments, each of which we modeled using the electric circuit analog shown in Figure 1: a capacitor captures the vascular pressure-volume relation, whereas resistance to blood flow is represented by linear resistances. The hydrostatic pressure source, $P_{\mathrm{h}}$, affects blood volume redistribution during changes in posture. We similarly represented the cardiac chambers but modeled their pumping action as time-varying capacitors. The en-

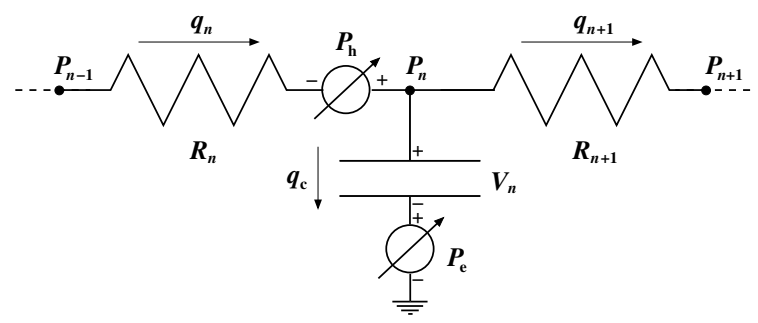

Figure 1. Single-compartment circuit representation. $P_{n-1}, P_{n}, P_{n+1}$ : compartment pressures; $P_{\mathrm{h}}, P_{\mathrm{e}}$ : hydrostatic and external pressures; $q_{n}, q_{n+1}, q_{c}$ : flow rates; $V_{n}$ : compartment volume; $R_{n}, R_{n+1}$ : flow resistance. 
tire hemodynamic system is closed-loop and contains four parallel peripheral vascular beds to allow for regionally distributed blood pooling.

We implemented set-point-based reflex models to represent the homeostatic action of the arterial baroreflex and the cardiopulmonary reflex [4]. The reflex models generate error signals by subtracting pre-defined pressure setpoints from pressure signals generated by the hemodynamic model. The error signals are subsequently convolved with impulse response functions and scaled by static gain values that are specific to the various effector mechanisms (heart rate, cardiac contractility, venous tone, and peripheral resistance).

\subsection{Simulations}

To validate the behavior of the cardiovascular model during orthostatic stress, we simulated the experimental protocol of Smith and co-workers [5], who subjected young and older male subjects to different degrees of headup tilt. We assessed the importance of mechanisms proposed to contribute to post-spaceflight OI by repeatedly simulating $75^{\circ}$ head-up tilts using a range of values for select parameters. We reduced total blood volume to simulate the effects of hypovolemia. We increased the venous compliance of the leg compartment to simulate the effects of increased capacity of the leg veins, and we simulated cardiac atrophy by reducing the right ventricular end-diastolic compliance. Finally, we simulated the effects of autonomic dysfunction by reducing the gain values to the arteriolar resistance and the venous tone reflex limbs. We quantified the impact of changes in these parameters on overall cardiovascular performance by measuring the change in arterial pressure in response to head-up tilts three minutes after the onset of stress. Experimental results and simulations are displayed as mean \pm standard error.

\section{Results}

Figure 2 shows the steady-state changes in mean arterial pressure (MAP, measured at heart level) and heart rate in response to tilts to various angles. The mean simulation is indicated by the solid lines; open circles represent data from young subjects, solid circles represent older subjects [5]. Both MAP and heart rate increase in response to graded orthostatic stress. The trends of the data are captured well by the simulations.

Figures $3-5$ show changes in MAP three minutes after the onset of a $75^{\circ}$ head-up tilt. In Figure 3 total blood volume is reduced in decrements of $2.5 \%$. The simulations indicate that the cardiovascular system is capable of maintaining MAP in the upright posture at or above supine baseline as long as the volume status is not too compromised. The cardiovascular system fails to protect MAP
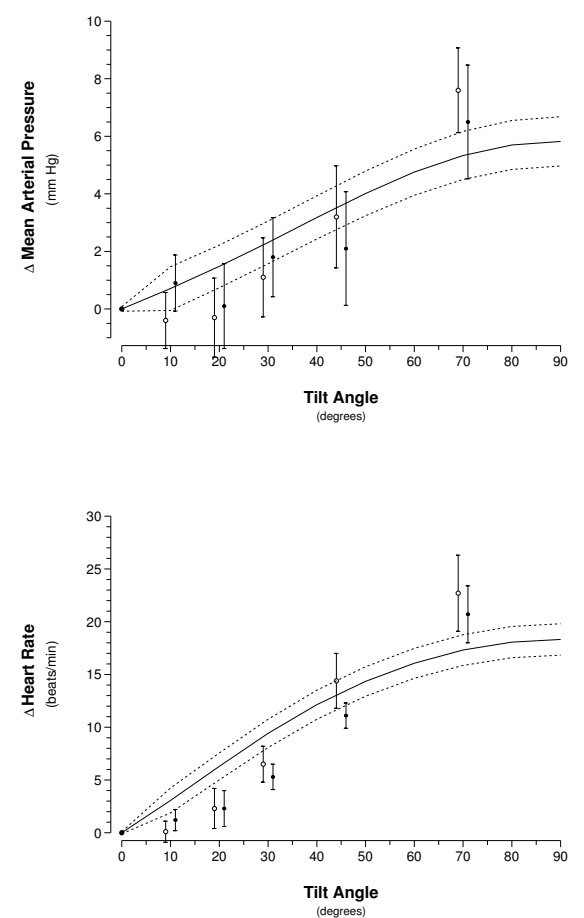

Figure 2. Change in mean arterial pressure (top) and heart rate (bottom) as a function of tilt angle. See text for details.

if reductions in total blood volume exceed 5\%. In contrast, detrimental changes of up to $15 \%$ in any of the other parameters only marginally impact the cardiovascular system's ability to protect MAP as indicated in Figure 4.

In Figure 5, we repeated the simulations shown in Figure 4 but started from a borderline hypovolemic volume status (5\% redcution in total blood volume). The parametric perturbations that previously impacted MAP only insignificantly now have serious consequences for maintaining arterial pressure in the upright posture.

\section{Discussion and conclusions}

Despite over forty years of experience in human space exploration, post-spaceflight OI remains a critical yet unresolved problem in the life-science space program. With current countermeasures in place, MAP in pre-syncopal astronauts can drop by more than $30 \mathrm{mmHg}$ when moving to the upright posture on landing day [2]. The simulations presented in the previous section aid in the understanding of the problem as they provide a possible explanation for the etiology of the syndrome.

The results displayed in Figures 3 and 4 suggest that from the mechanisms proposed, reductions in blood volume have the greatest detrimental impact on overall blood 


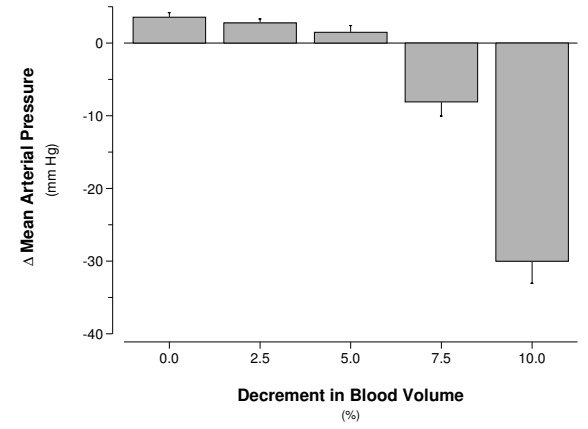

Figure 3. Dependence on volume status of mean arterial pressure response to $75^{\circ}$ head-up tilt.

pressure homeostasis in the upright posture. The results of Figure 4 indicate that if the volume status is not compromised, relatively large changes in any of the other parameters need to be hypothesized in order to account for the clinical picture seen post-flight. Reductions in total blood volume following spaceflight are highly variable; recent reports suggest a mean overall reduction of approximately $6 \%$ in male pre-syncopal astronauts [6]. Our simulations give credence to the view that hypovolemia is one of the principal contributors, yet at a level of $6 \%$ it might not be soly responsible for the clinical picture of the syndrome.

To assess the importance of mechanisms other than hypovolemia, we repeated the simulations of Figure 4 but superimposed the fractional changes in parameter values on a borderline hypovolemic volume status (5\% of hypovolemia). The results of this analysis are shown in Figure 5 and suggest that even moderate perturbations in any of the other parameters become very detrimental to arterial blood pressure homeostasis. Of particular importance are perturbations to the $\alpha$-sympathetically mediated reflex pathways of arteriolar resistance and venous tone, both of which depend on the peripheral neurotransmitter norepinephrine (NE). Meck and co-workers [7] have recently demonstrated that astronauts who become pre-syncopal on landing day fail to mount a significant vasoconstrictor response due to an inappropriately low release of $\mathrm{NE}$ in the upright posture. Our simulations suggest that in volumecompromised individuals failure to constrict arteries and veins leads to serious reductions in MAP in the upright posture. Furthermore, for similar fractional changes in gain values, reductions in venous tone gain impact MAP more dramatically than reductions in resistance gains.

In summary, our results point to possible etiologies of post-spaceflight OI based on either significant hypovolemia or a critical combination of hypovolemia and depressed vasoconstrictor capacity. In the absence of a functional fluid replacement countermeasure, an $\alpha$-adrenergic
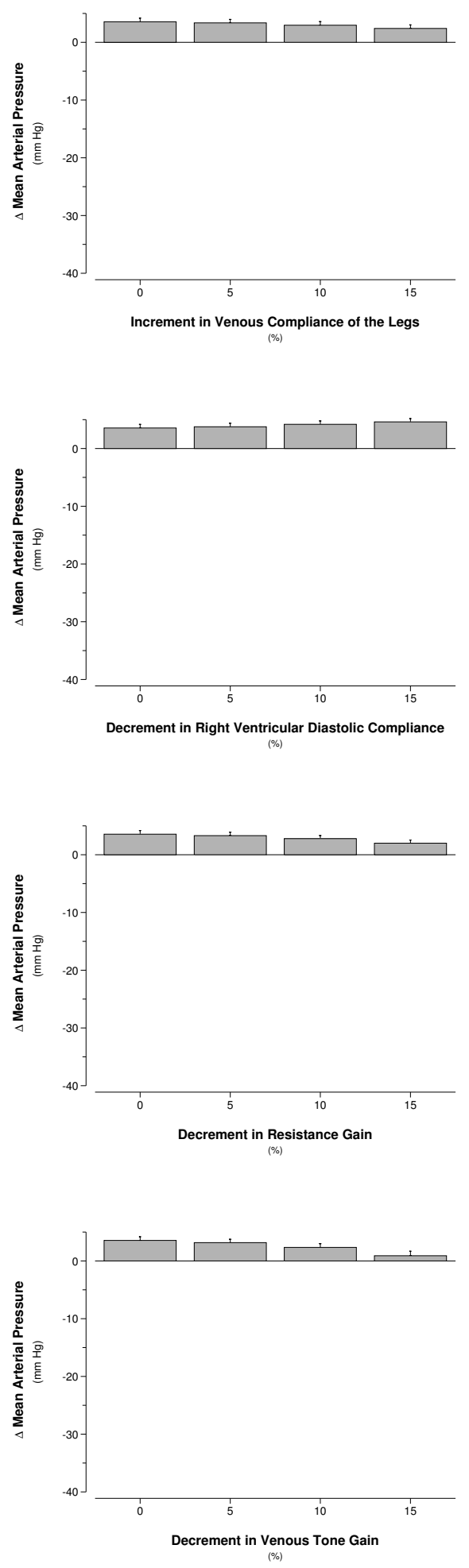

Figure 4. Mean arterial pressure response to $75^{\circ}$ head-up tilt under varying parametric conditions. Baseline volume status is euvolemic. 

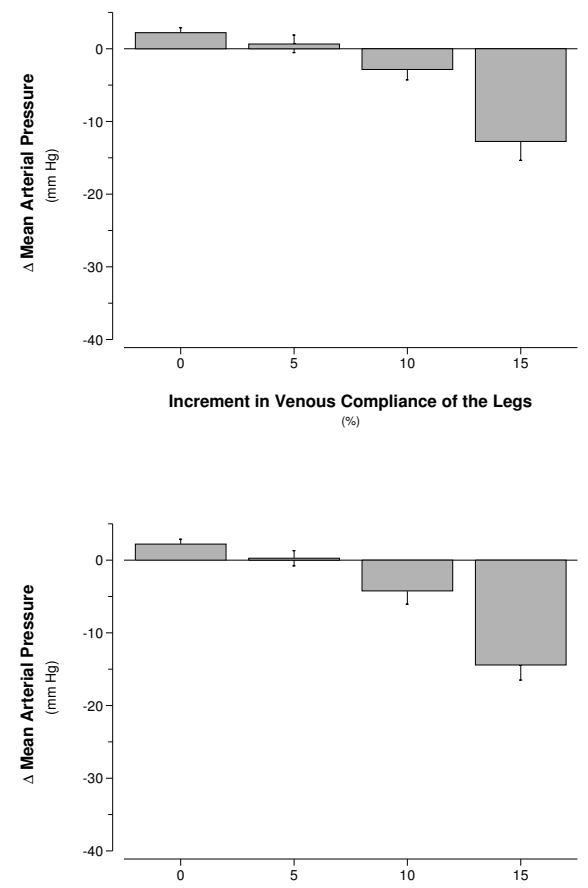

Decrement in Right Ventricular Diastolic Compliance

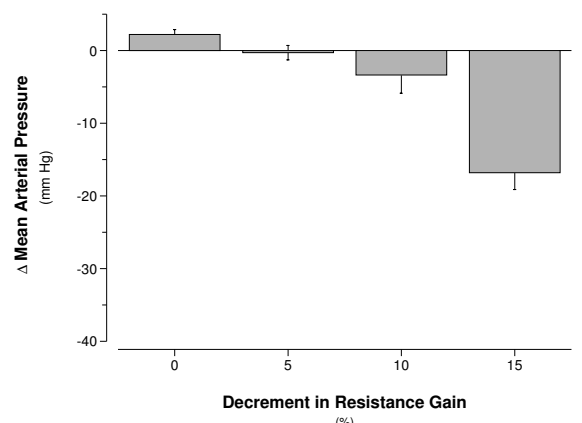

$(\%)$

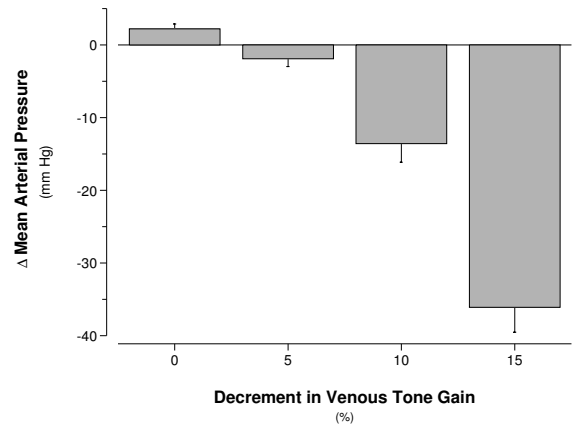

Figure 5. Mean arterial pressure response to $75^{\circ}$ head-up tilt under varying parametric conditions. Baseline volume status is hypovolemic. agonist, such as midodrine, might be a viable strategy to alleviate orthostatic symptoms post-flight. Initial success with midodrine supports this suggestion [8].

\section{Acknowledgements}

The National Aeronautics and Space Administration (NASA) supported this work through the NASA Cooperative Agreement NCC 9-58 with the National Space Biomedical Research Institute.

\section{References}

[1] Buckey J, Lane L, Levine J, Watenpaugh D, Wright S, Moore W, Gaffney F, Blomqvist C. Orthostatic intolerance after spaceflight. J Appl Physiol 1996;81(1):7-18.

[2] Meck J, Reyes C, Perez S, Goldberger A, Ziegler M. Marked exacerbation of orthostatic intolerance after long- vs. shortduration spaceflight in veteran astronauts. Psychosom Med 2001;63(6):865-873.

[3] Convertino V. Mechanisms of microgravity induced orthostatic intolerance: implications for effective countermeasures. J Gravit Physiol 2002;9(2):1-14.

[4] Heldt T, Shim E, Kamm R, Mark R. Computational modeling of cardiovascular response to orthostatic stress. J Appl Physiol 2002;92(3):1239-1254.

[5] Smith J, Hughes C, Ptacin M, Barney J, Tristani F, Ebert TJ. The effect of age on hemodynamic response to graded postural stress in normal men. Journal of Gerontology 1987; 42(4):406-411.

[6] Waters W, Ziegler M, Meck J. Postspaceflight orthostatic hypotension occurs mostly in women and is predicted by low vascular resistance. J Appl Physiol 2002;2(92):596-594.

[7] Meck J, Waters W, Ziegler M, deBlock H, Mills P, Robertson D, Huang P. Mechanisms of post-spaceflight orthostatic hypotension: $\alpha_{1}$-adrenergic receptor responses before flight and central autonomic dysregulation post-flight. Am J Physiol 2004;286(4):H1486-H1495.

[8] Platts S, Ziegler M, Waters W, Mitchell B, Meck J. Midodrine prescribed to improve recurrent post-spaceflight orthostatic hypotension. Aviat Space Enviro Med 2004; 75(6):554-556.

Address for correspondence:

Thomas Heldt, Ph.D.

MIT, Room 10-011

77 Massachusetts Avenue

Cambridge, MA 02139, USA

thomas@mit.edu 